\title{
Model-Based Estimation for Dynamic Cardiac Studies Using ECT
}

\author{
Ping-Chun Chiao, W. Leslie Rogers, Neal H. Clinthorne, Jeffrey A. Fessler, and Alfred O. Hero
}

\begin{abstract}
In this paper, we develop a strategy for joint estimation of physiological parameters and myocardial boundaries using ECT (Emission Computed Tomography). We construct an observation model to relate parameters of interest to the projection data and to account for limited ECT system resolution and measurement noise. We then use a maximum likelihood (ML) estimator to jointly estimate all the parameters directly from the projection data without reconstruction of intermediate images. We also simulate myocardial perfusion studies based on a simplified heart model to evaluate the performance of the modelbased joint ML estimator and compare this performance to the Cramer-Rao lower bound. Finally, we discuss model assumptions and potential uses of the joint estimation strategy.
\end{abstract}

\section{NOMENCLATURE}

$j$

$x$

$t$

$\tilde{y}_{i j}$

$y_{i j}$

$T_{i}$ $p(i \mid x)$

: detector position index

: time index

: emission position

: time

: observation of detected gamma rays at $i$-th detector position in $j$-th time interval

: mean observation at $i$-th detector position in $j$-th time interval

$: j$-th time interval

: system response function, i.e. the probability of detecting a gamma ray at $i$-th detector position given an emission at $x$

S : boundary parameter vector

$E_{u}(\mathbf{S}) \quad:$ emission space defined by left ventricular ROI

$E_{m}(\mathbf{S}) \quad$ : emission space defined by myocardial ROI

$E_{b}(\mathbf{S}) \quad$ : emission space defined by background region

$u(t) \quad$ : concentration function of left ventricular ROI

$m(t) \quad$ : concentration function of myocardial ROI

$b(t) \quad$ : concentration function of background region

Manuscript received September 22, 1991; revised August 10, 1993. This work was supported by the National Cancer Institute under Grant RO1CA32846 and by Squibb Diagnostics, Princeton, NJ. The associate editor responsible for coordinating the review of this paper and recommending its publication was R. Leahy.

The authors are with the Division of Nuclear Medicine, University of Michigan, Ann Arbor, MI USA.

IEEE Log Number 9401111.

$$
\begin{array}{ll}
u_{j}=\int_{T_{j}} u(t) d t & : \begin{array}{l}
j \text {-th time-integral concentration of left } \\
\text { ventricular ROI }
\end{array} \\
m(j)=\int_{T_{j}} m(t) d t: & j \text {-th time-integral concentration of } \\
& \text { myocardial ROI } \\
b_{j}=\int_{T_{j}} b(t) d t & : \begin{array}{l}
j \text {-th time-integral concentration of } \\
\text { background region }
\end{array} \\
\tilde{\mathbf{Y}} & : \text { vector concatenation of all } \tilde{y}_{i j} \\
\mathbf{Y} & : \text { vector concatenation of all } y_{i j} \\
\mathbf{\Theta} & : \text { vector concatenation of compartmental } \\
& \text { parameters } \\
\mathbf{H}(\boldsymbol{\Theta}) & : \text { matrix kernel characterizing the kinetics } \\
& \text { of myocardial ROI } \\
\mathbf{U} & : \text { vector concatenation of all } u_{i} \\
\mathbf{M} & : \text { vector concatenation of all } m_{j} \\
\mathbf{B} & : \text { vector concatenation of all } b_{j} \\
\mathbf{A} & =\left[\boldsymbol{\Theta}^{T} \mathbf{U}^{T} \mathbf{B}^{T}\right]^{T} \\
\mathbf{P} & =\left[\mathbf{A}^{T} \mathbf{S}^{T}\right]^{T}
\end{array}
$$

\section{INTRODUCTION}

$\mathrm{N}$ ADDITION to providing morphological information about imaged organs, a more powerful use of ECT (Emission Computed Tomography) is to quantify physiological and biological processes through dynamic imaging. In dynamic tomographic studies, one images an organ of interest over time to observe the dynamic behavior of the employed radiotracer. Ideally, the radiotracer is designed to measure specific physiological or biochemical processes. In steady state, the dynamic behavior of the radiotracer can usually be described by a linear compartmental model with constant compartmental parameters [1]. Often, these parameters are directly related to the specific process [2]. By tomographically measuring the organ tracer concentration (output), one can determine the compartmental parameters (system) using measurements of plasma tracer concentration (input). Dynamic imaging techniques have been widely used in PET (Positron Emission Tomography) quantification of myocardial blood flow [3], [4] and glucose metabolism [5] and have also been investigated in SPECT (Single Photon Emission Computed Tomography) cardiac studies [6].

In conventional dynamic data analysis, images indexed by time interval are reconstructed, and myocardial regions of interest (ROIs) are specified from the reconstructed images usually by human operators. A function of detected counts versus time index is calculated for each myocardial ROI. Along with plasma measurements, a parameter estimation algorithm is used to estimate compartmental parameters that 
underlie the concentration change in each ROI from the counttime function [3], [4].

Accurate quantification of functional processes with ECT is difficult to achieve because of two fundamental difficulties associated with ECT: limited system resolution and measurement noise (a combination of random emission and random absorption). In addition to the uncertainty in image values propagated from ECT measurement noise, limited system resolution results in a systematic error (bias) because of cross-contamination of ROI values between any two adjacent regions. In cardiac studies, several investigators have tried to correct this systematic error in myocardial ROI concentrations by assuming the myocardial wall thickness is known and precomputing the fraction of spill-over [7], or by formulating these fractions as unknown parameters [8]. Instead of postreconstruction correction, Carson [9] has generalized the pixelbased iterative image reconstruction to an ROI-based estimator based on maximum likelihood (ML) criteria. By incorporating a system response function to account for resolution blurring and by estimating ROI values directly from the projection data, he has shown the ROI-based ML estimator can generate statistically unbiased ROI concentration estimates (free from cross-contamination), if the ROIs are specified exactly. Carson [10] further formulated a parametric image model to include compartmental parameters. This formulation allows estimation of kinetic parameters directly from the projection data and can be written as follows

$$
\tilde{\mathbf{Y}} \sim \text { Poisson }\{\mathbf{W} \cdot \mathbf{X}(\boldsymbol{\Theta})\}
$$

In (1), $\tilde{\mathbf{Y}}$ is a vector concatenation of the projection data taken over time, $\mathbf{W}$ is a system response matrix to account for resolution blurring, and $\mathbf{X}(\Theta)$, parameterized by a compartmental parameter vector $\Theta$, is a pixel-based image vector. Snyder [11] and Ollinger [12] have also applied ROI-based ML estimators to compartmental parameter estimation using dynamic PET. Both post-reconstruction correction and ROIbased estimators assume that ROI delineation is exactly known from reconstructed ECT images.

In practice, the assumption of perfect ROI delineation is never true. The combination of image noise and poor resolution impairs one's ability to accurately delineate ROIs. Consequently, an extra source of error due to erroneous ROI specification is introduced into the estimation of ROI parameters. It is highly desirable to develop a strategy to reduce this error.

In this paper, we establish such a strategy through jointly estimating object boundaries as well as compartmental parameters. We construct an observation model to relate parameters of interest to the projection data and measurement noise. We then use an ML estimator to jointly estimate all the parameters directly from the projection data without reconstruction of intermediate images. Using a geometric model to characterize heart shape, we can parameterize not only compartmental parameters but also myocardial boundaries, left ventricular input function, and background concentration (lung, liver...etc.). The observation model we propose is more general than that in (1) and can be described as

$$
\tilde{\mathbf{Y}} \sim \operatorname{Poisson}\{\Psi(\mathbf{S}) \cdot \mathbf{C}(\boldsymbol{\Theta})\}
$$

where $\Psi$ characterizes imaging degradation factors and also depends on boundary parameters $\mathbf{S}$, and $\mathbf{C}$ is an ROI-based concentration vector and is parameterized by compartmental parameters $\Theta$.

In Section III below, we describe the observation model and a geometric model for the heart. In Section IV, we implement an ML estimator for use with these models. In Sections V and VI, we describe simulated myocardial perfusion studies using a polygonal heart model and a synthetic ECT system and show simulation results to demonstrate the performance of the model-based joint ML estimator (MJMLE). In Section VII we discuss simulation results and model assumptions. Finally, we conclude in Section VIII.

\section{OBSERVATION MODEL}

To simplify the following presentation, we make two assumptions. (1) On a short axis section the object model consists of three homogeneous regions: left ventricle, myocardium, and background. (2) The heart is stationary. These assumptions are further discussed in Section VII. This model is easily generalized to include multiple ROIs within the myocardium [13]. We start with a simple observation model as follows

$$
\tilde{\mathbf{Y}} \sim \operatorname{Poisson}\{\mathbf{Y}(\mathbf{P}, \text { System Response function })\}
$$

where $\mathbf{P}$ includes compartmental parameters $\boldsymbol{\Theta}$, myocardial boundaries $\mathbf{S}$, left ventricular concentrations $\mathbf{U}$, and background concentrations $\mathbf{B}$, and the system response function can account for resolution blurring, attenuation, random coincidence, deadtime, and scatter. We now describe the structure of $\mathbf{Y}$ by decomposing its element $y_{i j}$ at detector position $i$ and time $j$ as follows

$$
\begin{aligned}
y_{i j}= & \int_{T_{j}} u(t) d t \cdot \int_{E_{u}(\mathbf{S})} p(i \mid x) d x \\
& +\int_{T_{j}} m(t) d t \cdot \int_{E_{m}(\mathbf{S})} p(i \mid x) d x+\int_{T_{j}} b(t) d t \cdot \int_{E_{b}(\mathbf{S})} p(i \mid x) d x \\
= & u_{j} \cdot \int_{E_{u}(\mathbf{S})} p(i \mid x) d x+m_{j} \cdot \int_{E_{m}(\mathbf{S})} p(i \mid x) d x \\
& +b_{j} \cdot \int_{E_{b}(\mathbf{S})} p(i \mid x) d x
\end{aligned}
$$

Notice that in (4) the three integrals of the system response function are individually defined for the three assumed homogeneous regions. Similar to Huesman's approach [14], we approximate the myocardial concentrations $\mathbf{M}$ as functions of the left ventricular concentrations $\mathbf{U}$ and compartmental parameters $\Theta$

$$
\mathbf{M} \approx[(1-\alpha) \mathbf{H}(\boldsymbol{\Theta})+\alpha \mathbf{I}] \cdot \mathbf{U}=\mathbf{G}(\boldsymbol{\Theta}, \alpha) \cdot \mathbf{U}
$$

where I denotes identity matrix and $\alpha(0<\alpha<1)$ characterizes vascular space due to capillary bed in the myocardium. Although $\alpha$ can be jointly estimated with other parameters, for simplicity, we assume $\alpha$ is a known constant in the following discussions. From (4) and (5), a more compact formulation can be derived in a matrix form by collecting all the integrals 
in (4) in a matrix, $\Psi$, and concatenating all the concentration parameters as follows

$$
\mathbf{Y}(\mathbf{P})=\Psi(\mathbf{S}) \cdot\left[\begin{array}{c}
\mathbf{U} \\
\mathbf{G}(\mathbf{\Theta}) \cdot \mathbf{U} \\
\mathbf{B}
\end{array}\right]
$$

In searching for parameterization of the heart boundaries, we have been inspired by a procedure commonly used in cardiac ROI analysis. In most dynamic ECT cardiac studies, crosssectional, short-axis images are obtained by slicing a 3D heart image (constructed by interpolating a stack of reconstructed 2D heart images) perpendicular to the long axis of the heart. On these sliced images, the imaged heart is annulus-shaped and consists of a central region of left ventricle surrounded by myocardium. The right ventricular wall is thinner and tends not to show up on short-axis heart images. For this reason, we have treated the right ventricle wall as part of the background region. ROI analysis based on short-axis heart images always involves separation of the myocardial region from the left ventricular cavity, typically by drawing two closed curves around epicardium and endocardium. The shapes of the two curves are specified by multiple nodes with pre-specified angular separation. The nodes are individually allowed to move radially toward or away from the center of the curves. The final shapes of the two curves are individually obtained by connecting the two sets of nodes with piecewise smooth curves or straight lines (in this case, the heart shape is described by a polygon).

For simplicity, we choose to use a polygonal heart model. Generalization of this model is discussed in Section VII. By fixing a stationary point (the centroid) within the heart model, we formulate a linear combination of node radii as myocardial boundary parameters. Two node radii (for epicardial and endocardial nodes) are defined at each pre-specified angle with respect to the stationary point. In this manner, the position of each node in space is defined and, consequently, the backbone of the heart model is constructed by the epicardial and endocardial nodes. We subtract the epicardial radius from the endocardial radius at each angle and consider this difference as angular myocardial thickness. As a result, we parameterize the heart model by a set of epicardial radii and a set of angular thicknesses (both sets are concatenated in $\mathbf{S}$ ) with respect to a stationary point. The stationary point needs to be specified before estimation so that the boundary parameters can be defined. If the point is not appropriately chosen the first time, one can always adjust this stationary point after estimation and restart estimation with a new point.

We now explain in an intuitive way how these boundary parameters can be estimated. Careful examination of (6) reveals a simple relation as follows

$$
\operatorname{mass}(\mathbf{Y})=\operatorname{volume}(\Psi) \times \text { concentration }(\mathbf{C}) .
$$

If one considers the mean measurements $\mathbf{Y}$ as mass quantities then $\Psi$ must contain volume information. In particular, this volume information is characterized by the boundary parameters, and it specifies a portion from each ROI for each mass measurement to which this ROI contributes. Thus, boundary estimation is eventually accomplished through fitting a set of volumes and concentration parameters to the mass measurements.

\section{ESTIMATOR}

Given the observation model and model parameters defined in Section III, we can construct an ML estimator by implementing Fisher scoring, positivity constraints, and Marquardt's method [15] to jointly estimate all the parameters. Fisher scoring has been widely used in ML estimation in many situations. A thorough review of use of Fisher scoring in statistics can be found in [16]. It is similar in principle to Newton's method, which is an iterative process and is often used in solving nonlinear equations. Since the elements of $\mathbf{P}$ (compartmental parameters, concentrations, myocardial thicknesses, and endocardial radii) are physically positive, the ML estimate $\hat{\mathbf{P}}_{\text {ML }}$ of $\mathbf{P}$ is defined as

$$
\hat{\mathbf{P}}_{\mathrm{ML}} \equiv \arg \max _{\mathbf{P}} \ln f(\tilde{\mathbf{Y}} ; \mathbf{P}), \quad \mathbf{P}>0 .
$$

With the Poisson assumption for the measurement noise, the $\log$-likelihood $\ln f(\tilde{\mathbf{Y}} ; \mathbf{P})$ is given by

$$
\ln f(\tilde{\mathbf{Y}} ; \mathbf{P})=\sum_{i, j}\left(\tilde{y_{i j}} \cdot \ln y_{i j}(\mathbf{P})-y_{i j}(\mathbf{P})\right)+k
$$

where $k$ is a constant independent of $\mathbf{P}$. Obviously, $\ln f(\tilde{\mathbf{Y}} ; \mathbf{P})$ is not linear in $\mathbf{P}$, and $\hat{\mathbf{P}}_{\mathrm{ML}}$ cannot be solved directly. Fisher scoring suggests the following successive approximation

$$
\mathbf{P}^{n+1}=\mathbf{P}^{n}+\Delta \mathbf{P}^{n}, n=0,1 \ldots
$$

where

$$
\left.\Delta \mathbf{P}^{n} \equiv \mathbf{J}^{-1}(\mathbf{P}) \cdot \nabla_{\mathbf{P}} \ln f(\tilde{\mathbf{Y}} ; \mathbf{P})\right|_{\mathbf{P}=\mathbf{P}^{n}}
$$

When (10) converges in the $n$-th iteration based on prescribed criteria, the resulting $\mathbf{P}^{n+1}$ is considered as an approximation to $\hat{\mathbf{P}}_{\mathrm{ML}}$. In (11), J, the Fisher information matrix, approximates the Hessian matrix used in Newton's method [17] and can be derived for Poisson measurements as

$$
\begin{aligned}
\mathbf{J}(\mathbf{P}) & \equiv E\left(\left[\nabla_{\mathbf{P}} \ln f(\tilde{\mathbf{Y}} ; \mathbf{P})\right]\left[\nabla_{\mathbf{P}} \ln f(\tilde{\mathbf{Y}} ; \mathbf{P})\right]^{T}\right) \\
& =\nabla_{\mathbf{P}} \mathbf{Y}(\mathbf{P}) \cdot \operatorname{Diag}^{-1}\{\mathbf{Y}(\mathbf{P})\} \cdot\left[\nabla_{\mathbf{P}} \mathbf{Y}(\mathbf{P})\right]^{T}
\end{aligned}
$$

where $\operatorname{Diag}\{\mathbf{Y}(\mathbf{P})\}$ is a diagonal matrix containing the elements of $\mathbf{Y}(\mathbf{P})$ as its diagonal elements (see Appendix A for computation of the gradients).

To enforce $\hat{\mathbf{P}}_{\mathrm{ML}}>0$, we impose positivity constraints on each intermediate estimate $\mathbf{P}^{n}$. We choose to use the log transformation method [18] mainly because it provides computational convenience and is easy to implement. It can be shown that the log transformation gives the following iterative procedure

$$
\mathbf{P}^{n+1}=\mathbf{P}^{n} \cdot \exp \left(\operatorname{Diag}^{-1}\left\{\mathbf{P}^{n}\right\} \cdot \Delta \mathbf{P}^{n}\right), n=0,1 \ldots
$$

In (13), $\mathbf{P}^{n}$ is now forced to take on positive values for any positive initial estimate $\mathbf{P}^{0}$. Comparing (13) with (10), one can see that enforcing a positivity constraint using (13) only slightly increases computational complexity. 
TABLE I

MODEL CONFIgURATIONS, A SYTHETIC ECT SYSTEM, AND DATA SAMPLING SCHEMES

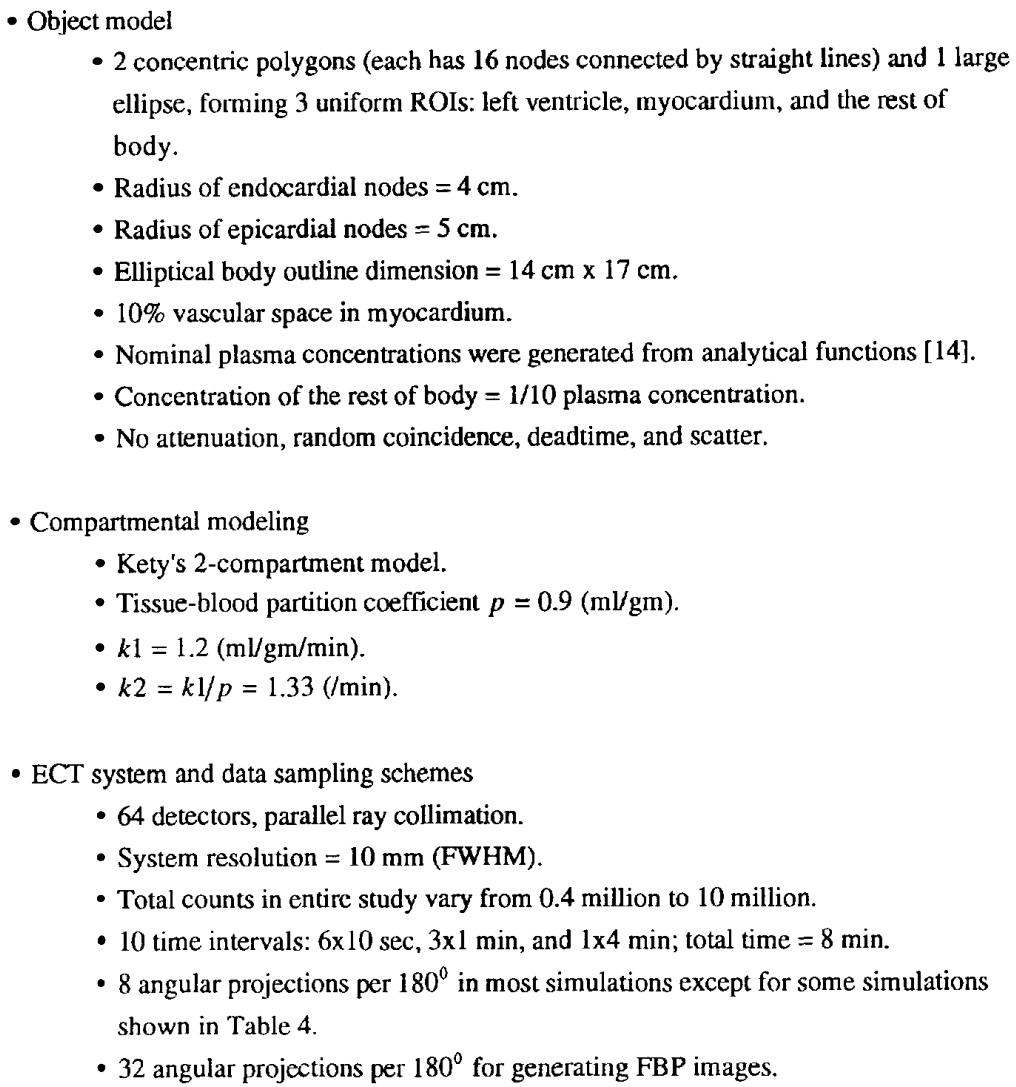

Using Fisher scoring alone may fail to converge to the maximum for the following reasons: (1) Iterative schemes based on Newton's method usually perform poorly and may not even converge, if they start in regions remote from the solution. (2) The approximation of the Hessian matrix by the Fisher information matrix may not be successful even in the neighborhood of the maximum, especially in case that the signal to noise ratio is low. We choose to implement a more general successive approximation scheme to optimally update $\Delta \mathbf{P}^{n}$, as suggested by Marquardt [15]. This method chooses the update direction $\Delta \mathbf{P}^{n}$ to lie in between the direction given by the Newton's iterative procedure and that given by the steepest ascent method. The latter is considered when the Newton's iterative procedure fails to increase the objective function (log-likelihood in our case).

\section{Simulation Study}

We have simulated myocardial perfusion studies for a freely diffusible tracer using a polygonal heart model and a synthetic ECT system in order to evaluate the performance of MJMLE, to quantify the improvement in accuracy of myocardial perfusion estimation over a fixed ROI analysis method in which myocardial boundaries are fixed at inaccurate values, and to determine the effects of model mismatches. In the fixed ROI analysis method, myocardial boundary (node radii) delineation errors were simulated from independent and identically distributed (IID) Gaussian distributions with $1.5 \mathrm{~mm}$ standard deviation (SD), and the perfusion and left ventricular input function were estimated directly from projection data. We show MJMLE is practically unbiased for count levels exceeding 1 million. We evaluate the performance by comparing the root-mean-square (RMS) error of MJMLE estimates with the Cramer-Rao (CR) lower bound defined by the diagonal elements of the inverse of the Fisher information matrix. When the RMS error of the estimates is close to the square root of the lower bound, we claim the estimator practically achicves the lower bound. We also present a set of boundary estimates in image format to demonstrate the robustness of the estimator for a variety of initial boundary conditions and to show the convergence pattern of boundary estimates. To address the issue of model mismatches, we simulate projection data based on a circular heart model and estimate perfusion using a polygonal model.

Table I summarizes model configurations, a synthetic ECT system, and data sampling schemes. We have used an object 


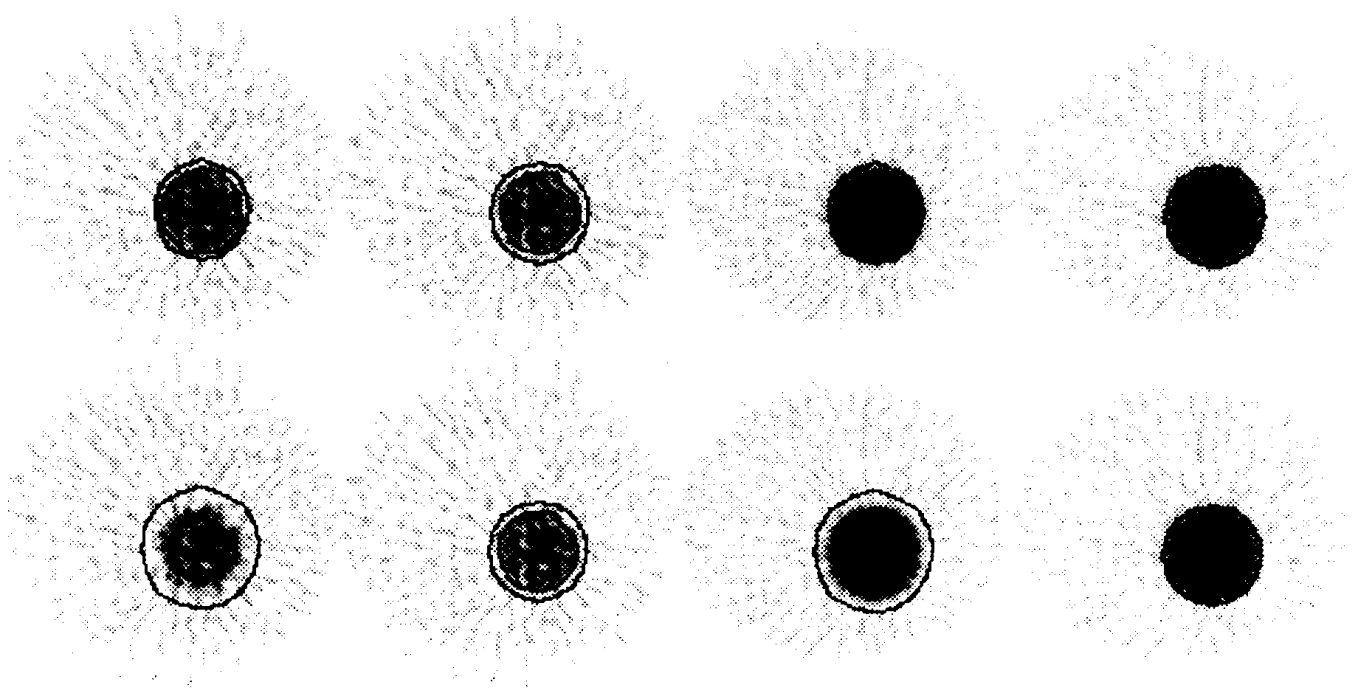

Fig. 1. A set of boundary estimates superimposed on FBP images corresponding to two different time intervals. The first column shows initial boundary estimates that either underestimate (upper image) or overestimate (lower image) myocardial thickness. The second column illustrates final boundary estimates that are visually identical despite the dramatically different initial conditions. The four identical images in the first two columns are from the second time interval, in which left ventricular counts dominate the signal (showing low contrast at the epicardial boundary). The images in the last two columns, superimposed on the same set of boundary estimates, are from the last time interval, in which left ventricular concentrations and myocardial concentrations have similar magnitudes (showing lower contrast at the endocardial boundary).

model that consists of two parts: a heart, and the rest of body. The body contour was assumed known. We modeled the heart using a stationary polygonal model (the issue of heart motion is discussed in Section VII). Heart boundaries were parameterized by a set of epicardial node radii and a set of angular thicknesses of the myocardium with respect to the centroid of the model. Myocardial perfusion $(k 1)$ was modeled by Kety's 2-compartment model [19], [20]. Estimates of the left ventricular concentrations were used to estimate the input function using a linear interpolator [14]. To facilitate computation, we have chosen to use a depth-independent system response function so that a convenient convolution operation can be applied to rapidly compute projection data, as described in [21]. To reduce the total computation time for the multiple realizations, we have simulated data sets with only eight projections over $180^{\circ}$. To demonstrate the validity of this small system approximation, we have also compared the CR lower bound for using sampling schemes with more projections (Table IV).

The statistics of parameter estimates were computed using 200 to 300 realizations. Counting noise was generated using a Poisson distribution based on mean projection data. In most simulations, mean projection data were computed based on the same polygonal model that was used in estimation, except when examining the effects of model mismatches, where a circular model was used. In all simulations, initial boundary estimates (node radii with respect to the centroid) were randomly generated from IID Gaussian distributions with $1.5 \mathrm{~mm}$ SD. Other initial parameter estimates were also generated from independent Gaussian distributions with SD of $10 \%$ of their nominal values.
Iterations were stopped when the following conditions were satisfied:

$$
\begin{aligned}
0 & \leq \\
& \frac{\ln f^{n+1}-\ln f^{n}}{\ln f^{n}}<10^{-6} \text { and } \\
& 2\left|p_{i}^{n}-p_{i}^{n+1}\right|<10^{-4}\left(\left|p_{i}^{n}\right|+\left|p_{i}^{n+1}\right|\right), \forall i
\end{aligned}
$$

where $f^{n}$ is the log-likelihood and $p_{i}^{n}$ is the $i$-th parameter estimate during the $n$-th iteration. The estimation process usually converges within 10 iterations at 1 million counts or above. Each iteration takes less than a minute to complete on a micro VAX III workstation.

\section{RESULTS}

\section{A. Estimation Performance}

Table II lists the statistics of a set of simulation results for every parameter in $\mathbf{P}$ except background concentration parameters. The total counts in this entire study equal 0.4 million. Overall, the RMS error of the estimates is close to the lower bound in each case. While the RMS errors of input estimates vary with time, those of boundary estimates are quite uniform. The estimated bias values in $k 1$ and $k 2$ are less than $0.7 \%$ and those in boundary parameters are less than $0.3 \mathrm{~mm}$.

\section{B. Robustness}

Fig. 1 presents a set of boundary estimates superimposed on filtered-back projection (FBP) images corresponding to 2 different time intervals. The first column shows initial boundary estimates that either underestimate (upper image) or overestimate (lower image) myocardial thickness. The second column illustrates final boundary estimates that are visually 
TABLE II

STATISTICS OF A SET OF Simulation RESUltS (TOTAL COUNTS IN ENTIRE STUDY $=0.4$ MILLION, NUMBER OF REALIZATIONS $=228$ )

\begin{tabular}{|c|c|c|c|c|c|}
\hline & Parameter & Bias & SD & RMS & ower bound \\
\hline & $k 1$ & $-.68 \pm .41(\%)$ & $6.19 \pm .15(\%)$ & $6.23 \pm .15(\%)$ & $6.23(\%)$ \\
\hline & $k 2$ & $-.63 \pm .42$ & $6.29 \pm .15$ & $6.32 \pm .15$ & 6.18 \\
\hline $10 \mathrm{sec}$ & Input function & $.56 \pm .13(\%)$ & $1.92 \pm .05(\%)$ & $2.00 \pm .05(\%)$ & $1.88(\%)$ \\
\hline $20 \mathrm{sec}$ & estimated from & $.44 \pm .09$ & $1.36 \pm .03$ & $1.43 \pm .03$ & 1.35 \\
\hline $30 \mathrm{sec}$ & left ventricular & $.49 \pm .1$ & $1.47 \pm .03$ & $1.55 \pm .04$ & 1.53 \\
\hline $40 \mathrm{sec}$ & concentrations & $-.16 \pm .15$ & $2.31 \pm .05$ & $2.32 \pm .05$ & 2.16 \\
\hline $50 \mathrm{sec}$ & in 10 time & $-.06 \pm .18$ & $2.65 \pm .06$ & $2.65 \pm .06$ & 2.66 \\
\hline $60 \mathrm{sec}$ & intervals & $-.18 \pm .19$ & $2.82 \pm .07$ & $2.83 \pm .07$ & 2.78 \\
\hline $120 \mathrm{sec}$ & & $-.15 \pm .07$ & $1.12 \pm .03$ & $1.13 \pm .03$ & 1.14 \\
\hline $180 \mathrm{sec}$ & & $-.20 \pm .07$ & $1.05 \pm .02$ & $1.07 \pm .03$ & 1.09 \\
\hline $240 \sec$ & & $.10 \pm .07$ & $1.12 \pm .03$ & $1.13 \pm .03$ & 1.05 \\
\hline $480 \mathrm{sec}$ & & $.01 \pm .04$ & $.61 \pm .01$ & $.61 \pm .01$ & 6 \\
\hline 1 & Radii for 16 & $-.14 \pm .15(\mathrm{~mm})$ & $2.20 \pm .05(\mathrm{~mm})$ & $2.21 \pm .05(\mathrm{~mm})$ & $2.43(\mathrm{~mm})$ \\
\hline 2 & endocardial & $.05 \pm .15$ & $2.26 \pm .05$ & $2.26 \pm .05$ & 2.43 \\
\hline 3 & nodes & $-.16 \pm .16$ & $2.47 \pm .06$ & $2.48 \pm .06$ & 2.43 \\
\hline 4 & & $-.20 \pm .15$ & $2.21 \pm .05$ & $2.22 \pm .05$ & 2.44 \\
\hline 5 & & $.24 \pm .17$ & $2.50 \pm .06$ & $2.51 \pm .06$ & 2.44 \\
\hline 6 & & $-.17 \pm .17$ & $2.58 \pm .06$ & $2.59 \pm .06$ & 2.45 \\
\hline 7 & & $-.13 \pm .16$ & $2.46 \pm .06$ & $2.46 \pm .06$ & 2.45 \\
\hline 8 & & $.04 \pm .17$ & $2.52 \pm .06$ & $2.52 \pm .06$ & 2.45 \\
\hline 9 & & $-.13 \pm .17$ & $2.48 \pm .06$ & $2.48 \pm .06$ & 2.46 \\
\hline 10 & & $-.22 \pm .16$ & $2.45 \pm .06$ & $2.46 \pm .06$ & 2.46 \\
\hline 11 & & $.10 \pm .16$ & $2.45 \pm .06$ & $2.46 \pm .06$ & 2.46 \\
\hline 12 & & $-.11 \pm .17$ & $2.51 \pm .06$ & $2.51 \pm .06$ & 2.47 \\
\hline 13 & & $-.11 \pm .15$ & $2.28 \pm .05$ & $2.28 \pm .05$ & 2.48 \\
\hline 14 & & $-.17 \pm .15$ & $2.26 \pm .05$ & $2.26 \pm .05$ & 2.46 \\
\hline 15 & & $.22 \pm .16$ & $2.34 \pm .05$ & $2.35 \pm .06$ & 2.46 \\
\hline 16 & & $-.06 \pm .15$ & $2.22 \pm .05$ & $2.22+.05$ & 244 \\
\hline 1 & Myocardial & $.20 \pm .16(\mathrm{~mm})$ & $2.39 \pm .06(\mathrm{~mm})$ & $2.40 \pm .06(\mathrm{~mm})$ & $2.56(\mathrm{~mm})$ \\
\hline 2 & thicknesses & $-.04 \pm .16$ & $2.41 \pm .06$ & $2.41 \pm .06$ & 2.56 \\
\hline 3 & measured & $.17 \pm .17$ & $2.63 \pm .06$ & $2.63 \pm .06$ & 2.55 \\
\hline 4 & along 16 & $.20 \pm .16$ & $2.34 \pm .05$ & $2.34 \pm .05$ & 2.57 \\
\hline 5 & node radii & $-.21 \pm .18$ & $2.64 \pm .06$ & $2.65 \pm .06$ & 2.57 \\
\hline 6 & & $.16 \pm .18$ & $2.71 \pm .06$ & $2.71 \pm .06$ & 2.58 \\
\hline 7 & & $.22 \pm .17$ & $2.61 \pm .06$ & $2.62 \pm .06$ & 2.58 \\
\hline 8 & & $-.09 \pm .18$ & $2.70 \pm .06$ & $2.71 \pm .06$ & 2.58 \\
\hline 9 & & $.20 \pm .17$ & $2.61 \pm .06$ & $2.62 \pm .06$ & 2.59 \\
\hline 10 & & $.25 \pm .17$ & $2.59 \pm .06$ & $2.60 \pm .06$ & 2.59 \\
\hline 11 & & $-.08 \pm .17$ & $2.55 \pm .06$ & $2.55 \pm .06$ & 2.59 \\
\hline 12 & & $.09 \pm .17$ & $2.61 \pm .06$ & $2.61 \pm .06$ & 2.61 \\
\hline 13 & & $.16 \pm .16$ & $2.43 \pm .06$ & $2.43 \pm .06$ & 2.61 \\
\hline 14 & & $.20 \pm .16$ & $2.41 \pm .06$ & $2.42 \pm .06$ & 2.59 \\
\hline 15 & & $-.30 \pm .17$ & $2.51 \pm .06$ & $2.53 \pm .06$ & 2.58 \\
\hline 16 & & $.09 \pm .16$ & $2.34 \pm .05$ & $2.34 \pm .05$ & 2.57 \\
\hline
\end{tabular}

and numerically identical despite the dramatically different initial conditions. In fact, perfusion and other parameter estimates are also numerically identical in this case. The four identical images in the first two columns are from the second time interval, in which left ventricular counts dominate the signal (showing low contrast at the epicardial boundary). The images in the last two columns, superimposed on the same set of boundary estimates, are from the last time interval, in which left ventricular concentrations and myocardial concentrations have similar magnitudes (showing lower contrast at the endocardial boundary). Fig. 2 shows the iteration sequence (from left to right, top to bottom) that takes the initial boundary estimates illustrated in the lower first image in Fig. 1, which overestimate the thickness by roughly $2 \mathrm{~cm}$, to the final estimates shown in the lower second image in Fig. 1.

\section{Perfusion Estimation}

Fig. 3 plots percent-bias in myocardial perfusion estimates as a function of total counts in the entire study (for a single slice). The bias in perfusion estimates decreases from $0.7 \%$ to near $0.0 \%$ as the number of counts increases from 0.4 to 10 million counts. Fig. 4 compares the performance of MJMLE and the fixed ROI analysis method with the lower bound for $k 1$. MJMLE nearly achieves the lower bound for $k 1$ over a range of signal strength between 0.4 and 10 million counts. The fixed ROI analysis method, on the other hand, gives worse performance in comparison with the lower bound as the number of counts increases; the RMS error of $k 1$ estimates is 4 times higher than the lower bound at 10 million counts.

\section{Model Mismatch}

Table III compares the systematic error associated with boundary model mismatches. The oversimplified 8-node model used for data generated from a circular model introduces $4 \%$ bias in $k 1$ estimates, while the 16-node model (used in most of our simulations) shows no significant increase in the bias in comparison with the case where the data model and 


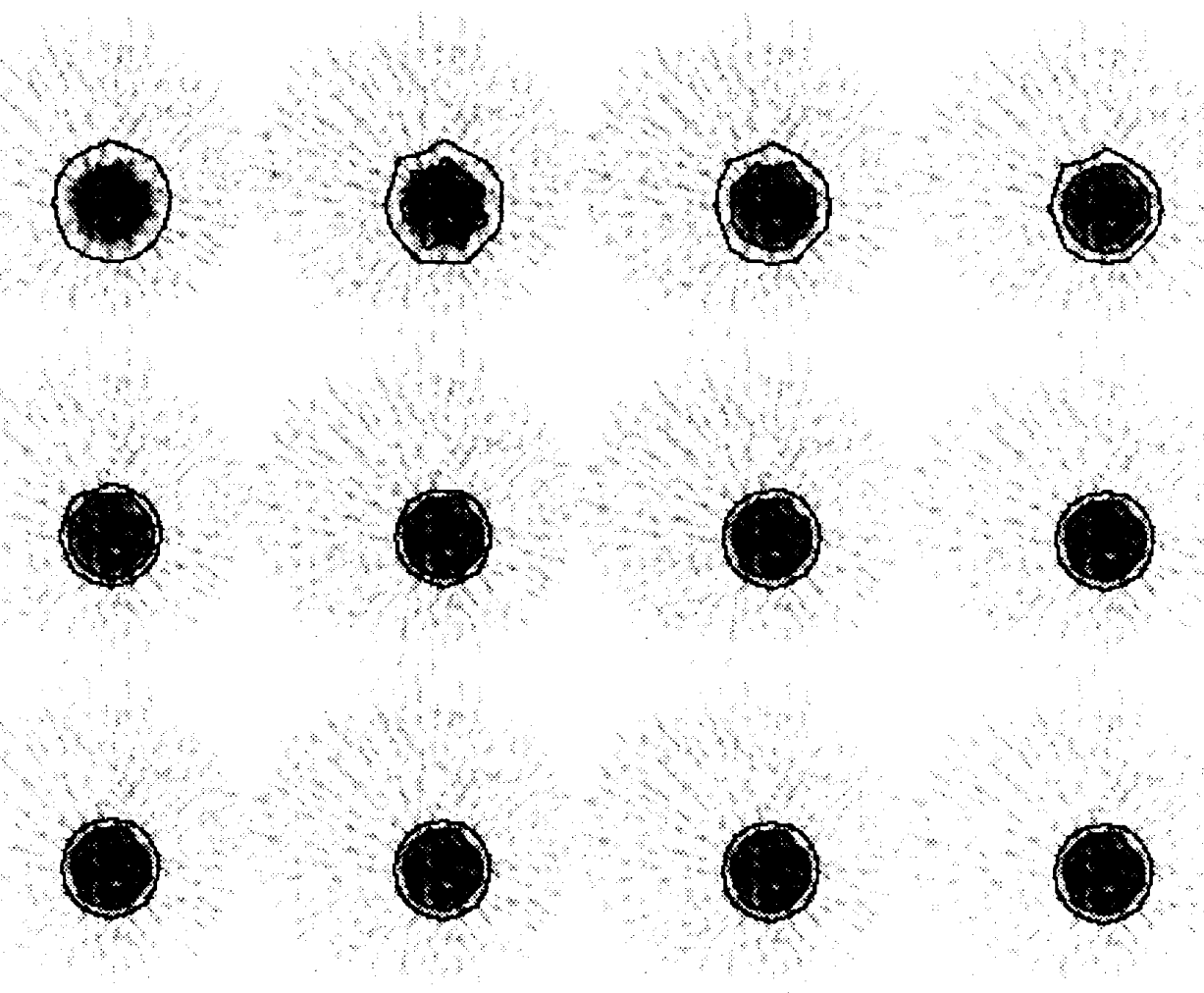

Fig. 2. The iteration sequence (from left to right, top to bottom) that takes the initial boundary estimates illustrated in the lower first image in Fig. 1, which overestimate the thickness by roughly $2 \mathrm{~cm}$, to the final estimates shown in the lower second image in Fig. 1 .

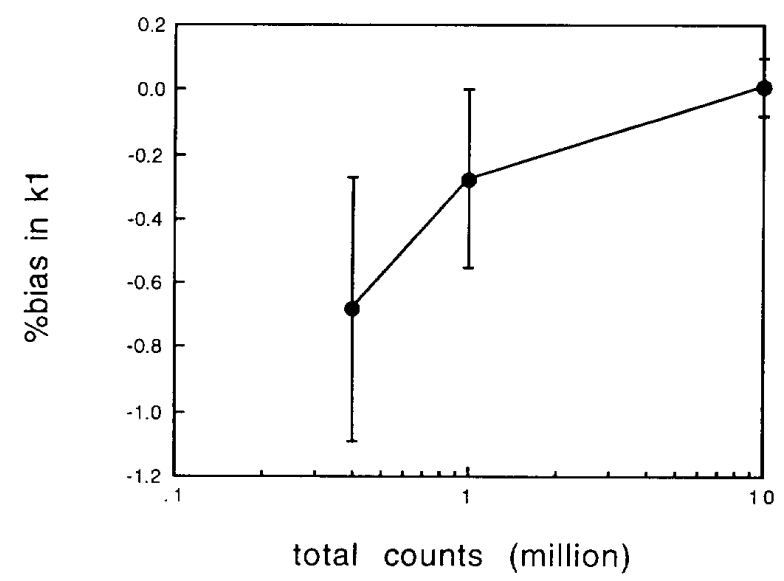

Fig. 3. Percent-bias in myocardial perfusion estimates as a function of total counts (number of realizations $=228,213$, or 200 at $0.4,1$, or 10 million counts, respectively). The percent-bias in estimates decreases as the number of counts increases.

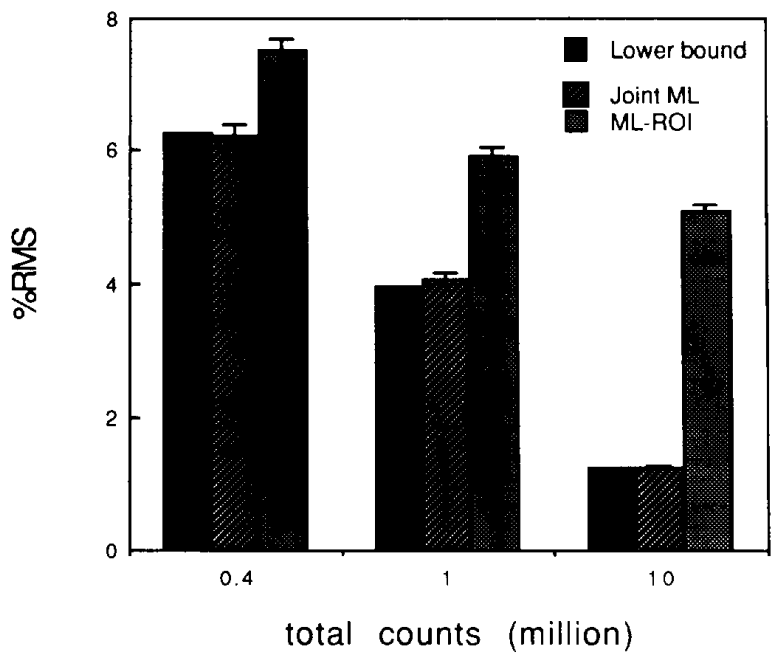

Fig. 4. Performance comparison of MJMLE and the fixed ROI analysis method with the lower bound for $k 1$ (number of realizations $=228,213$, or 200 for MJMLE and 200, 210, or 200 for fixed ROI method at $0.4,1$, or 10 million counts). MJMLE nearly achieves the lower bound for perfusion over a range of signal strength between 0.4 and 10 million counts. The fixed ROI analysis method, on the other hand, gives worse performance in comparison with the lower bound as the number of counts increases; the RMS error of k:1 estimates is four times higher than the lower bound at 10 million counts. 
TABLE III

COMPARISON OF BIAS ASSOCIATED WITH MODEL MISMATCHES (TOTAL COUNTS IN ENTIRE STUDY $=1$ MILLION, NUMBER OF REALIZATIONS $=300,222$, OR 213 FOR THE FOLLOWING THREE CASES, RESPECTIVELY)

\begin{tabular}{|c|c|c|c|}
\hline & $\begin{array}{l}>8 \text {-node } \\
>\text { circular }\end{array}$ & $\begin{array}{l}16 \text {-node } \\
\text { circular }\end{array}$ & $\begin{array}{c}\text { 16-node } \\
\text { 16-node polygonal }\end{array}$ \\
\hline$k 1$ & $-3.90 \pm .21(\%)$ & $-.35 \pm .26(\%)$ & $-.28 \pm .28(\%)$ \\
\hline Radii of & $1.95 \pm .04(\mathrm{~mm})$ & $.48 \pm .1(\mathrm{~mm})$ & $-.15 \pm .1(\mathrm{~mm})$ \\
\hline endocardial & $2.04 \pm .04$ & $.57 \pm .1$ & $.12 \pm .09$ \\
\hline nodes & $2.00 \pm .04$ & $.37 \pm .1$ & $-.05 \pm .1$ \\
\hline (actual value & $1.98 \pm .04$ & $.37 \pm .1$ & $-.03 \pm .1$ \\
\hline $4 \mathrm{~cm})$ & $1.96 \pm .04$ & $.55 \pm .1$ & $-.04 \pm .11$ \\
\hline & $1.98 \pm .04$ & $.59 \pm .1$ & $-.12 \pm .11$ \\
\hline & $1.91 \pm .04$ & $.62 \pm .1$ & $.15 \pm .1$ \\
\hline & $1.96 \pm .04$ & $.39 \pm .1$ & $-.08 \pm .1$ \\
\hline & & $.52 \pm .1$ & $.00 \pm .11$ \\
\hline & & $.46 \pm .1$ & $-.01 \pm .1$ \\
\hline & & $.49 \pm .1$ & $.06 \pm .1$ \\
\hline & & $.73 \pm .1$ & $-.18 \pm .1$ \\
\hline & & $.45 \pm .11$ & $.00 \pm .1$ \\
\hline & & $.52 \pm .1$ & $.10 \pm .1$ \\
\hline & & $.40 \pm .1$ & $.01 \pm .1$ \\
\hline & & $61+.1$ & $-.01 \pm .11$ \\
\hline Angular & $1.15 \pm .04(\mathrm{~mm})$ & $.16 \pm .1(\mathrm{~mm})$ & $.16 \pm .11(\mathrm{~mm})$ \\
\hline thicknesses of & $1.04 \pm .04$ & $.10 \pm .11$ & $-13 \pm .1$ \\
\hline myocardium & $1.09 \pm .04$ & $.23 \pm .1$ & $.03 \pm .11$ \\
\hline (actual value & $1.13 \pm .04$ & $.36 \pm .1$ & $.02 \pm .11$ \\
\hline $1 \mathrm{~cm})$ & $1.15 \pm .04$ & $.06 \pm .11$ & $.04 \pm .12$ \\
\hline & $1.12 \pm .05$ & $.07 \pm .11$ & $.08 \pm .11$ \\
\hline & $1.20 \pm .04$ & $.01 \pm .1$ & $-.09 \pm .11$ \\
\hline & $1.14 \pm .04$ & $.25 \pm .11$ & $.04 \pm .11$ \\
\hline & & $.12 \pm .11$ & $.06 \pm .11$ \\
\hline & & $.17 \pm .11$ & $-.02 \pm .11$ \\
\hline & & $.09 \pm .11$ & $-.06 \pm .11$ \\
\hline & & $-.02 \pm .11$ & $.22 \pm .11$ \\
\hline & & $.19 \pm .11$ & $-.02 \pm .1$ \\
\hline & & $.08 \pm .11$ & $-.12 \pm .1$ \\
\hline & & $.30 \pm .11$ & $-.02 \pm .11$ \\
\hline & & $.00 \pm .1$ & $.04 \pm .11$ \\
\hline
\end{tabular}

TABLE IV

COMPARISON OF SQUARE-ROOT LOWER BOUNDS FOR DIFFERENT SAMPLING SCHEMES (TOTAL COUNTS IN ENTIRE STUDY = 1 MILLION)

\begin{tabular}{lccc} 
& 8 angular projections & 16 angular projections & 32 angular projections \\
\hline $\begin{array}{l}\text { k1 } \\
\begin{array}{l}\text { Mean radius of } \\
\text { endocardial } \\
\text { nodes (mean) }\end{array}\end{array}$ & $1.94(\%)$ & $3.94(\%)$ & $3.94(\%)$ \\
$\begin{array}{l}\text { Mean angular } \\
\text { thickness } \\
\text { of myocardium } \\
\text { (mean) }\end{array}$ & $1.63(\mathrm{~mm})$ & $1.5(\mathrm{~mm})$ & $1.5(\mathrm{~mm})$ \\
\hline
\end{tabular}

observation model are consistent (fourth column). In both inconsistent cases (8-node and 16-node), MJMLE tends to overestimate boundary parameters to compensate for smaller regions that are enclosed by observation models with the same radii as used in data models.

\section{E. Sampling}

Table IV compares the lower bounds for different tomographic sampling schemes. Eight angular projections, which are used in most of our simulations, result in nearly identical lower bounds as compared to the other much better sampling schemes. This is due to the low dimensional parameterization of the parameters of interest.

\section{DISCUSSION}

Results in Table II demonstrate that MJMLE nearly achieves the lower bounds at 0.4 million counts, with biases in all parameters less than $1 \%$. This count density (for a single slice) is usually considered a low signal to noise situation in ECT measurements. Thus, the results imply that MJMLE may be very useful in dynamic cardiac studies. The percentbias plot in Fig. 3 shows that MJMLE is also asymptotically unbiased in perfusion estimation and that acceptably small biases are achieved at experimentally achievable count rates. The randomness in initial boundary estimates $(\mathrm{SD}=1.5 \mathrm{~mm})$ is perhaps an optimistic characterization of ROI delineation error. Even so, as shown in Fig. 4, joint estimation of perfusion and boundaries clearly shows significant improvement over 
the fixed ROI analysis method, especially in high count rate situations.

From Table III, one sees that a 16-node heart model is adequate for describing a circular $2 \mathrm{D}$ short axis heart slice. The polygonal model is easy to use and can be easily upgraded for better precision simply by increasing the number of nodes. Furthermore, the polygonal structure can be conveniently extended to model a 3D heart. Thus, for cardiac studies, it may not be necessary to use more complicated surface interpolators such as splines [22] and Fourier descriptors [23], [24].

More nodes mean more parameters and, therefore, more degrees of freedom in parameter estimates. Thus, one expects to see higher variance in node radius estimates with an increase in the number of nodes. In fact, if one wants to estimate more than 16 nodes at 0.4 million counts, boundary regularization methods should be included [13]. We have occasionally obtained angular thickness estimates near zero using a 16-node model at 0.2 million counts. This is not hard to understand from a predicted SD of almost $4 \mathrm{~mm}$ in angular thickness estimates at 0.2 million counts.

Using Kety's 2-compartment model, we have simulated a situation similar to a PET $\mathrm{H}_{2}^{15} \mathrm{O}$ perfusion study, in which contrast is so poor that additional measurements are usually required to delineate the left ventricular region [3]. The images shown in Fig. 1 have typical characteristics of low contrast as often seen on $\mathrm{H}_{2}^{15} \mathrm{O}$ perfusion images. It is evident from the boundary estimates shown in Fig. 1 that the joint estimation strategy outperforms what can possibly be done by visual delineation. It is also apparent from an average RMS error of $1.6 \mathrm{~mm}$ in myocardial boundary estimates at 1 million counts and $0.5 \mathrm{~mm}$ at 10 million counts that the model-based estimation has exceeded the limit of system resolution $(10 \mathrm{~mm}$ FWHM in this case). This should not be surprising because MJMLE has in fact included system blurring, counting statistics, geometric modeling, and input-output (left ventricular input function-myocardial concentrations) modeling.

Although our model is based on two simplified assumptions (homogeneous regions and stationary heart), the model can be readily extended to account for regional inhomogeneity and cardiac motion. One can segment the myocardial ROI into a number of smaller ROIs in which the homogeneity assumption is more appropriate. For the usually large background region, since it is not of primary interest, instead of further segmentation, one may introduce a set of weights to account for regional inhomogeneity. It can be shown [21] that such weights take the following form

$$
\int_{E_{b}(\mathbf{S})} p(i \mid x) d x \cdot \frac{V_{b}(\mathbf{S}) \cdot \int_{T_{j}} \lambda_{b}(x, t) d t}{\int_{E_{b}(\mathbf{S}) \cdot \int_{T_{j}} \lambda_{b}\left(x^{\prime}, t^{\prime}\right) d t^{\prime} d x^{\prime}}} d x
$$

where $\lambda_{b}(x, t)$ is the radiotracer distribution function of the background region at emission position $x$ and time $t$ and $V_{b}(\mathbf{S})$ is the total volume of the background region. The idea of using such weights, although formulated differently, has been proposed by Ollinger [25]. Although $\lambda_{b}(x, t)$ is unknown in practice, one can conceivably approximate $\lambda_{b}(x, t)$ by a series of reconstructed emission images. In fact, Ollinger has suggested such an approximation [25] as in his words: "The tracer activity distribution, which, being both unknown and nonstationary, must be approximated by a series of reconstructed images.... These time intervals must be long enough to ensure that the images contain enough counts to be a good estimate of the tracer activity distribution, but short enough to compensate for the nonstationarity of the underlying process." Alternatively, one can also introduce a set of low order basis functions (sinusoidal functions, for instance) to characterize the regional inhomogeneity and jointly estimate the coefficient for each basis function.

Our model-based approach directly applies to gated studies, which have been proposed to deal with cardiac motion [26]. In gated studies, each cardiac cycle is timed and divided into $\mathrm{K}$ time frames and counting measurements are acquired and partitioned into these $\mathrm{K}$ gated groups. For such studies, one simply introduces additional boundary parameters for each set of gated measurements. Of course, in these situations, the data in each time interval are much noisier and additional model constraints on boundaries will likely be required.

Applying the joint estimation strategy to other organs will require appropriate boundary models that are clearly taskdependent. The model-based joint estimator can potentially be configured for tumor studies and studies of other organs where ROIs are isolated and have simple shapes. One key requirement for successful use of a geometric model is to prevent the model from degenerating in each estimation iteration. This degeneration, if not carefully considered, could happen, for instance, in an estimation iteration in which epicardial and endocardial boundaries cross each other. As demonstrated in our polygonal heart model, we have proposed to use a stationary point and to define boundary parameters that are physically positive with respect to this point. In this manner, we have successfully overcome model degeneration by enforcing the constraint $\mathbf{P}^{n}>0$ in each estimation iteration.

In general, the iteration processes defined in either $(10)$ or (13) do not guarantee convergence to the global maximum for a nonlinear model as in (6). In the simplified simulations, we have found that MJMLE is fairly robust against the random initialization tested $(1.5 \mathrm{~mm}$ SD for the initial boundary estimates and SD of $10 \%$ of the nominal values for other parameters). In practice, one can randomize initial estimates over a physiological range of parameter values to verify the local behavior of the estimation processes.

Although the simulations do not include attenuation, random coincidence, deadtime, and scatter, these effects can be readily accounted for in the system response function in (3) and (4) by using measured survival probability, random coincidence rates, deadtime compensation, and scatter response function, respectively. Due to the random nature of these measurements, we expect a loss in estimation performance when the measurements are used in MJMLE.

\section{CONCLUSION}

We have developed a model-based strategy for joint estimation of compartmental parameters and myocardial boundaries and have constructed a MJMLE for use with this strategy. 
We have also demonstrated in simplified simulation studies that MJMLE is almost statistically efficient for the various experimental conditions tested. Although the simulations show promising results, further experimental verifications and investigations on issues such as geometric modeling, regional inhomogeneity, and cardiac motion are required before this estimation strategy can be used in clinical applications.

\section{APPENDIX A}

The iterative estimation processes defined in (10) and (13) require computing the gradient of the log-likelihood. In particular, as shown in (11) and (12), this computation involves computing the following term

$$
\nabla_{\mathbf{P}} \mathbf{Y}(\mathbf{P})=\left[\begin{array}{c}
\nabla_{\mathbf{A}} \mathbf{Y}(\mathbf{P}) \\
\nabla_{\mathbf{S}} \mathbf{Y} \mathbf{P}
\end{array}\right]
$$

Usually, $\nabla_{\mathbf{A}} \mathbf{Y}(\mathbf{P})$ can be analytically derived. However, $\nabla_{\mathbf{S}} \mathbf{Y}(\mathbf{P})$ cannot be obtained analytically. Using (7) $\nabla_{\mathbf{S}} \mathbf{Y}(\mathbf{P})$ is given as

$$
\nabla_{\mathbf{S}} \mathbf{Y}(\mathbf{P})=\left[\begin{array}{c}
\frac{\partial}{\partial s_{1}} \Psi(\mathbf{S}) \cdot \mathbf{C}(\mathbf{A}) \\
\frac{\partial}{\partial s_{2}} \Psi(\mathbf{S}) \cdot \mathbf{C}(\mathbf{A}) \\
\cdot \\
\cdot \\
\frac{\partial}{\partial s_{n}} \Psi(\mathbf{S}) \cdot \mathbf{C}(\mathbf{A})
\end{array}\right]
$$

where $n$ is equal to $2 \times$ number of nodes, $s_{m}$ is the $m$ th element of $\mathbf{S}$, and the nonzero elements of $\frac{\partial}{\partial s_{m}} \Psi(\mathbf{S})$ have the form

$$
\frac{\partial}{\partial s_{m}} \int_{E_{k}(\mathbf{R})} p(i \mid x) d x
$$

If a pixelated system response $p(i \mid l) \equiv \int_{l \text { th pixel }} p(i \mid x) d x$ is available, (A.3) can be approximated by

$$
\frac{1}{\Delta s_{m}}\left(\sum_{l}\left(\mathbf{I}_{k}\left(\mathbf{S}+\Delta s_{m} \cdot \mathbf{e}_{m}, l\right)-\mathbf{I}_{k}(\mathbf{S}, l)\right) \cdot p(i \mid l)\right)
$$

where $\mathbf{e}_{m}$ is the $m$-th unit vector and $\mathbf{I}_{k}(\mathbf{S}, l)$ denotes an indicator function that is 0 for pixels not in the $k$-th region, 1 for pixels totally in the region, and between 0 and 1 for pixels partially in the region.

\section{REFERENCES}

[1] J. A. Jacquez, Compartmental Analysis in Biology and Medicine. 2nd ed. Ann Arbor: The University of Michigan Press, 1988.

[2] M. E. Phelps, J. C. Mazziotta, and H. R. Schelbert, Positron Emission Tomography and Autoradiography Principles and Applications for the Brain and Heart. New York: Raven Press, 1986.

[3] S. R. Bergmann, P. Herrero, J. Markham, C. J. Weinheimer, and M. N. Walsh. "Noninvasive quantitation of myocardial blood flow in human subjects with oxygen-15-labeled water and positron emission tomography," J. Amer. Coll. Cardiol., vol. 14, pp. 639-652, 1989.
[4] G. D. Hutchins, M. Schwaiger, K. C. Rosenspire, J. Krivokapich, H. Schelbert, and D. E. Kuhl, "Noninvasive quantification of regional blood flow in the human heart using $\mathrm{N}-13$ ammonia and dynamic positron emission tomographic imaging," J. Amer. Coll. Cardiol, vol. 15, pp. 1032-1042, 1990 .

[5] S. S. Gambhir, M. Schwaiger, S. C. Huang, J. Krivokapich, H. R. Schelbert, C. A. Nienaber, and M. E. Phelps, "Simple noninvasive quantification method for measuring myocardial glucose utilization in humans employing positron emission tomography and fluorine-18 deoxyglucose," J. Nucl. Med., vol. 30, pp. 359-366, 1989.

[6] A. M. Smith, G. T. Gullberg, F. L. Datz, and P. E. Christian, "Kinetic modeling of teboroxime using dynamic SPECT imaging," J. Nucl. Med., vol. 33, p. 878, 1992.

[7] E. Henze, S. C. Huang, O. Ratib, E. Hoffman, M. E. Phelps, and H. R Schelbert, "Measurements of regional tissue and blood-pool radiotracer concentrations from serial tomographic images of the heart," $J$. Nucl. Med, vol. 24, pp. 987-996, 1983.

[8] P. Herrero, J. Markham, and S. R. Bergmann, "Quantitation of myocardial blood flow with $\mathrm{H} 215 \mathrm{O}$ and positron emission tomography: assessment and error analysis of a mathematical approach," J. Comput. Assist. Tomogr., vol. 13, pp. 862-873, 1989.

[9] R. E. Carson, "A maximum likelihood method for region-of-interest evaluation in emission tomography," J. Comput. Assist. Tomogr., vol. 10, pp. 654-663, 1986

[10] R. E. Carson and K. Lange, "The EM parametric image reconstruction algorithm," J. Amer. Statist. Assoc., vol. 80, pp. 20-22, 1985.

[11] D. L. Snyder, "Parameter estimation for dynamic studies in emissiontomography systems having list-mode data," IEEE Trans. NuCl. Sci. vol. NS-31, pp. 925-931, 1984.

[12] J. M. Ollinger, "Estimation algorithms for dynamic tracer studies using positron-emission tomography," IEEE Trans. Med. Imag., vol. 6, pp. $115-125,1987$.

[13] P. Chiao, W. L. Rogers, J. A. Fessler, N. H. Clinthorne, and A. O. Hero, "Model-based estimation with boundary side information or regularization," IEEE Trans. Med, Imag., vol. 13. no. 2, pp. 227-234, June 1994.

[14] R. H. Huesman and B. M. Mazoyer, "Kinetic data analysis with a noisy input function," Phys. Med. Biol, vol. 32, pp. 1569-79, 1987.

[15] D. W. Marquardt, "An algorithm for least-squares estimation of nonlinear parameters," J. Soc. Indust. Appl. Math., vol. 11, pp. 431-441, 1963.

[16] P. J. Green, "Iteratively reweighted least squares for maximum likelihood estimation and some robust and resistant alternatives," J. Roy. Statist. Soc, vol. 46, pp. 149-192, 1984.

[17] W. H. Press, B. P. Flannery, S. T. Teukolsky, and W. T. Vetterling Numerical Recipes. Cambridge, U.K.: Cambridge University Press, 1988

[18] R. Fietcher, Practical Methods of Optimization. 2nd. ed. New York: John Wiley \& Sons, 1987.

[19] S. S. Kety, "Theory of blood-tissue exchange and its application to measurement of blood flow," Methods Med. Res., vol. 8, pp. 223-236, 1960

[20] S. S. Kety, "Measurement of local blood flow by the exchange of an inert, duffusible substance," Methods Med. Res., vol. 8. pp. 228-236, 1960.

[21] P. Chiao, "Parameter estimation strategies for dynamic cardiac studies using emission computed tomography," Ph.D. dissertation, University of Michigan, 1991.

[22] R. H. Bartels, H. C. Beatty, and B. A. Barsky, An Intoduction to Splines for Use in Computer Graphics and Geometric Modeling. New York: Morgan Kaufmann Publishers, 1987.

[23] A. Jouan, "Analysis of sequences of cardiac contours by Fourier descriptors for plane closed curves," IEEE Trans. Med. Imag., vol. 6 , pp. 176-180, 1987

[24] C. T. Zahn and R. Z. Roskies, "Fourier descriptors for plane closed curves," IEEE Trans. Comput., vol. C-21, pp. 269-281, 1972.

[25] J. M. Ollinger and D. L. Snyder, "An evaluation of an improved method for computing histograms in dynamic tracer studies using positronemission tomography," IEEE Trans. Nucl. Sci., vol. NS-29, pp. 474-478, 1986

[26] E. J. Hoffman, M. E. Phelps, G. Wisenberg, H. R. Schelbert, and D. E. Kuhl, "Electrocardiographic gating in positron emission computed tomography," J. Comput. Assist. Tomogr., vol. 3, pp. 733-9, 1979. 